This work was supported by grants from the National Key Technology Research and the Development Program for 12th Five-Year Plan (No. 2011BAI11B20) and Technology Integration Innovation Project of Shaanxi Province (2013KTCL03-01).

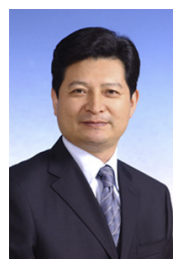

\section{Reply to the Editor:}

We appreciate Ying-Fu Chen and colleagues for their interest in our article, ${ }^{1}$ and we are delighted with their thoughtful insights into our results. We also thank the Editor for the possibility to reply.

Previous studies have shown the clinical features and difference of acute aortic dissection (AAD) between China and other countries. ${ }^{2}$ However, it is necessary to keep in mind that the previous studies in China were sporadic rather than multicenter registered research. To better understand the current situation of patients with aortic dissection in China and the differences between China and developed countries, the Registry of Aortic Dissection in China (Sino-RAD), which is the first registry system in China designed to provide an unbiased representative population of patients with $\mathrm{AAD}$, was established including 15 large cardiovascular centers. The preliminary investigation disclosed the differences between Sino-RAD and the International Registry of Acute Aortic Dissection (IRAD), as shown in the article. Of note, the ratio of patients with hypertension was lower than in other reports from China., This may be due to a partially different database. Another interesting finding is the lower in-hospital mortality of type A AAD. This may be due to the group of younger patients and new "standard" therapy for type A dissection, which is based on morphologic characteristics of Chinese people. ${ }^{5}$

However, as mentioned in our article, the IRAD data and Sino-RAD data are at different periods. In addition, most of the Sino-RAD data are from Chinese Han people, which cannot reflect Asian people. With the development of medical technology, the disease shows different features at different times. There may be a certain degree of bias to conclude the differences between Sino-RAD and IRAD. We have to admit that this is a great limitation of the article. The real differences need to be clarified by using the same period database. This is what we are trying to do next. We are trying to conduct a common research with IRAD, German Registry for Acute Aortic Dissection Type A, and other databases. As the search goes on, more detailed research data will be illustrated, such as characteristic of type A AAD, type B AAD, intramural hematoma, and so forth. The optimal approach would be to make a thorough evaluation of AAD.

\section{Weiguang Wang, $M D$ \\ Weixun Duan, $M D$ \\ Dinghua Yi, MD \\ on behalf of the Registry of Aortic Dissection in China}

(Sino-RAD) investigators

Department of Cardiovascular Surgery

Xijing Hospital

The Fourth Military Medical University

Xi'an, Shaanxi, P. R. China

\section{References}

1. Wang W, Duan W, Xue Y, Wang L, Liu J, Yu S, et al. Clinical features of acute aortic dissection from the Registry of Aortic Dissection in China. J Thorac Cardiovasc Surg. 2014;148:2995-3000.

2. Wang DJ, Fan FD, Wang Q, Li QG, Zhou Q, Wu Z, et al. Preliminary characterization of acute aortic dissection in the mainland of China. Chin Med J (Engl). 2011;124:1726-30.

3. Li Y, Yang N, Duan W, Liu S, Yu S, Yi D. Acute aortic dissection in China. Am J Cardiol. 2012;110:1056-61.

4. Ma WG, Zheng J, Zhang W, Sun K, Ziganshin BA, Wang LF, et al. Frozen elephant trunk with total arch replacement for type A aortic dissections: does acuity affect operative mortality? J Thorac Cardiovasc Surg. 2014;148:963-72.

5. Sun L, Qi R, Zhu J, Liu Y, Zheng J. Total arch replacement combined with stented elephant trunk implantation: a new "standard" therapy for type a dissection involving repair of the aortic arch? Circulation. 2011;123:971-8.

\section{http://dx.doi.org/10.1016/j.jtcvs.2015.03.006}

\section{THE STICH TRIAL DATA: KEEP IT SIMPLE}

\section{To the Editor:}

Elegance is simplicity; confusion is complexity. The Surgical Treatment for Ischemic Heart Failure (STICH) Trial investigators have reported another analysis focusing on viability. ${ }^{1}$ One thousand patients were randomly allocated to undergo coronary artery bypass grafting (CABG) or CABG plus surgical ventricular reconstruction (SVR) for anterior wall severe left ventricular dysfunction. They concluded that assessment of myocardial viability by single-photon emission computed tomography did not discriminate patients who would profit from the addition of SVR.

Several deficiencies of the STICH trial must be reviewed. Only 276 of the 1000 enrolled patients had a viability test, a central requirement for inclusion in the original study design. Holly and colleagues ${ }^{1}$ report that 46 of these patients $(17 \%)$ had anterior or apical viability and were randomly allocated to CABG or CABG plus SVR. Why was SVR done in these patients? They should have been excluded. SVR was done because of visual regional dysfunction assessed intraoperatively. Unsurprisingly, there was no difference in outcome. Maybe this was hibernating ventricle. Clearly this was not a group for SVR, as reported by numerous surgical registries.

The big news from this new study compares patients with anterior scar who underwent $\mathrm{CABG}$ versus $\mathrm{CABG}$ plus SVR. No difference in outcomes was noted. Preoperative volumes are reported, and they are large, but lacking is the crucial measurement of postoperative volume. Previous STICH data showed a left ventricular reduction of $19 \%$ in 